\title{
Efecto de diferentes relaciones de luz azul:roja en el crecimiento de plántulas de chile habanero (Capsicum chinense Jacq.)
}

\author{
Effect of different blue:red light ratios on habanero pepper seedlings \\ (Capsicum chinense Jacq.) growth
}

\author{
Mendoza-Paredes J.E*, Castillo-González A.M', Valdéz-Aguilar L.A², Avitia-García E , García-Mateos M.R \\ Departamento de Fitotecnia, Universidad Autónoma Chapingo. Km 38.5 Carretera México-Texcoco. CP. 56230, Texcoco de \\ Mora, Estado de México, México. \\ Departamento de Horticultura, Universidad Autónoma Agraria Antonio Narro. Calzada Antonio Narro 1923. CP. 25315, \\ Buenavista, Saltillo, Coahuila, México.
}

\section{RESUMEN}

El chile habanero es una de las especies hortícolas de mayor importancia en la península de Yucatán y la producción de plántula es la parte crucial inicial para una producción exitosa de frutos. Estas se podrían cultivar en ambiente controlado con iluminación LED y permitiría producirse en cualquier época del año libres de plagas y enfermedades. Los objetivos fueron evaluar el efecto de diferentes proporciones de luz LED azul(A):roja(R) (A0\%, A34.5\%:R65.5\%, A57.1\%:R42.9\%, A76\%:R24\%, A:100\%) y un testigo fluorescente sobre crecimiento de planta y concentración de pigmentos fotosintéticos en plántulas de chile habanero 'Mayan Ba'alché' bajo condiciones controladas de crecimiento. Los resultados mostraron que el testigo promovió el crecimiento de la hoja, a través de una mayor área foliar, índice de área foliar, área foliar específica, peso fresco de hoja y con una menor frecuencia estomática y diámetro de tallo, mientras el tratamiento A100\% promovió el crecimiento del tallo, a través de una mayor altura de planta, distancia de entrenudos y peso fresco de tallo. Los tratamientos A34.5\%:R65.5\%, A57.1\%:R42.9\%, A76\%:R24\% indujeron un efecto negativo en altura de plántula, distancia de entrenudos y peso fresco de tallo. Los tratamientos no tuvieron efecto en peso seco de parte aérea ni en pigmentos fotosintéticos.

Palabras clave: Luz LED, luz fluorescente, concentración de pigmentos fotosintéticos, ambiente controlado

\section{ABSTRACT}

Habanero pepper is one of the most important horticultural species in the Yucatan peninsula, and the seedling production is the crucial initial part for successful fruit production. These could be grown under controlled environment with LED lighting and would allow them to be produced all year-round, free of pests and diseases. The objectives were to evaluate the effects of different blue(A):red(R) LED light ratios (A0\%, A34.5\%:R65.5\%, A57.1\%:R42.9\%, A76\%:R24\%, A:100\%) and a fluorescent control on plant growth and photosynthetic pigments concentration of habanero pepper seedlings 'Mayan Ba'alché' grown under controlled conditions. The results showed that control promoted leaf growth, through a greater leaf area, leaf area index, specific leaf area, leaf fresh weight and with a lower stomatal frequency and stem

*Autor para correspondencia: Jorge Enrique Mendoza Paredes Correo electrónico:jmenpar07@outlook.com

Recibido: 7 de abril de 2021

Aceptado: 26 de diciembre de 2021 diameter, while A100 \% treatment promoted stem growth, through greater plant height, internode distance and stem fresh weight. Treatments A34.5\%:R65.5\%, A57.1\%:R42.9\%, A76\%:R24\% induced a negative effect in seedling height, internode distance and stem fresh weight. The treatments had no effect on dry weight of aerial part or in photosynthetic pigments.

Key words: LED light, fluorescent light, photosynthetic pigments concentration, controlled environment.

\section{INTRODUCCIÓN}

El chile habanero es una planta anual de 30 a $120 \mathrm{~cm}$ de altura, de tipo arbusto (Lim, 2013), es nativa de las tierras bajas de la cuenca amazónica, de donde se dispersó en la época prehispánica a Perú, a la cuenca del Orinoco, las Antillas y finalmente arribó a la Península de Yucatán (Trujillo, 2018). Es una de las especies hortícolas de mayor importancia de la península de Yucatán (Ruiz-Lau et al., 2011; Castillo-Aguilar et al., 2015) y obtuvo la denominación de origen en esta región en 2010 (CICY, 2016). El chile habanero posee diversas propiedades nutraceúticas, medicinales, e industriales, lo que lo hace tener una alta demanda en el mercado nacional e internacional (Adame-García et al., 2021; Lim, 2013).

La producción de plántula de chile habanero es la parte crucial inicial para una posterior producción exitosa de frutos, es la etapa donde más vulnerable es a plagas, enfermedades y variaciones de condiciones ambientales, y tiene un periodo que abarca de los 40 a 50 días después de la siembra (Castillo-Aguilar et al., 2015).

Un sistema de producción con el cual se puede obtener plántulas de manera más uniforme y libre de riesgos ambientales, biológicos y/o nutricionales es el uso de la agricultura vertical en ambiente controlado con iluminación LED, este un sistema de producción agrícola cerrado y aislado del ambiente circundante mediante el uso de estructuras sólidas y herméticas, adicionalmente se utiliza una fuente de iluminación LED en sustitución de la luz solar para el crecimiento de las plantas y un sistema de aire acondicionado, donde en conjunto se puede controlar, establecer y monitorear todas las variables ambientales, nutricionales y de riego necesarias en una planta a través de un equipo eléctrico-mecánico controlado por software especializado y en el cual las plan- 
tas se colocan para su crecimiento en una estructura tipo anaquel con múltiples niveles, con lo cual se logra tener un aumento de producción basado en el número de niveles que se utilicen en una superficie determinada (Kozai y Niu, 2016; Mendoza-Paredes et al., 2021 b).

La producción en este sistema permite obtener plántulas fisiológica y morfológicamente uniformes a pesar de las condiciones climáticas de la región (Kozai et al., 2016), además no se presenta estacionalidad en la producción del cultivo y en consecuencia puede ser producida en cualquier época del año (Mendoza-Paredes et al., 2021a). Adicionalmente, se puede acortar el periodo de producción de plántulas entre un $30 \%$ a $40 \%$ en comparación con las que se producen en condiciones de invernadero con alta tecnología en control de variables ambientales, nutrición mineral, plagas y enfermedades (Kozai et al., 2016).

Las plántulas producidas de esta manera se encuentran libres de plagas y enfermedades (Kozai et al., 2016), por lo tanto, no se necesita la aplicación de ningún tipo de producto de prevención y/o control de estas (Mendoza-Paredes et al., 2021a).

La producción de plántula de chile habanero es idónea para cultivarse con agricultura vertical en ambiente controlado con iluminación LED (Kozai y Niu, 2016), ya que además la plántula de chile habanero tiene un ciclo corto de crecimiento y posee un porte relativamente pequeño (Castillo-Aguilar et al., 2015).

Actualmente no existe investigación de esta especie en condiciones controladas de crecimiento con luz LED. Se reportan investigaciones en condiciones similares con plántulas de tomate (Hernández et al., 2016; Kim y Hwang, 2019; Liu et al., 2018; Wollaeger y Runkle, 2015) y en plántula de pepino por Hernández y Kubota (2016), donde cada investigación se obtuvieron efectos diferentes para cada especie y variedad en relación a diferentes proporciones de luz LED roja y azul.

El objetivo del presente estudio fue evaluar el efecto de diferentes proporciones de luz LED roja y azul sobre crecimiento y concentración de pigmentos fotosintéticos en hojas de plántula de chile habanero.

\section{MATERIALES Y MÉTODOS Germinación}

Se utilizaron semillas de chile habanero 'Mayan Ba'alché' del CICY, las cuales se sembraron en charolas, con un tamaño de cavidad de $3.1 \mathrm{~cm} \times 3.1 \mathrm{~cm}$ (base superior), 6.0 $\mathrm{cm}$ (altura), $2.1 \mathrm{~cm} \times 2.1 \mathrm{~cm}$ (base inferior) y $23 \mathrm{~mL}$ (volumen), una semilla por cavidad a una profundidad de $1 \mathrm{~cm}$ en una mezcla de turba:perlita (3:1) [turba rubia (Pro $\mathrm{mix}^{\circledR}$ ) y perlita $\left(\right.$ Agrolita $\left.{ }^{\oplus}\right)$ ], las cuales se cortaron para tener 80 cavidades (4 filas $\times 20$ columnas). Una vez sembradas las semillas, las charolas se colocaron en condiciones de laboratorio donde tuvieron luz solar incidente durante el día y fueron regadas con agua potable (pH 7.1 y CE $\left.0.42 \mathrm{dS} \mathrm{m}^{-1}\right)$. La temperatura ambiental (día $25.8 \pm 0.2^{\circ} \mathrm{C} /$ noche $22.6 \pm 0.1^{\circ} \mathrm{C}$ ) y la humedad relativa (día $51.5 \pm 0.6 \% /$ noche $58.6 \pm 0.6 \%$ ), se registraron con un datalogger Extech instruments ${ }^{\circledR}$ modelo RH10. A los 12 días después de la emergencia bajo estas condiciones, se llevaron a una cámara de crecimiento para iniciar los tratamientos.

\section{Cámara y gabinetes de crecimiento}

Se realizó una adaptación de una cámara de crecimiento a partir de un cuarto frío usado (TORREY® ${ }^{\circledR}, \mathrm{MEX}$ ), con medidas interiores de $2.76 \mathrm{~m} \times 2.76 \mathrm{~m} \times 1.90 \mathrm{~m}$ (ancho, largo y altura), las modificaciones internas de la cámara de crecimiento se basó en el diseño de Katagiri et al. (2015), con las cuales se reguló la temperatura ambiental y la humedad relativa a través de un aire acondicionado portátil (LG ${ }^{\circledR}$ modelo LP1017WSR 10200 BTU, KOR) y un deshumidificador (Whirlpool ${ }^{\circledR}$ modelo WAD200 5Q 20L/24 h, USA) los cuales se colocaron dentro de la cámara de crecimiento.

Se diseñaron seis gabinetes de crecimiento con la finalidad de mantener aislados los tratamientos de luz. Éstos se construyeron a partir de un anaquel metálico $(0.3 \mathrm{~m}$ de ancho $\times 0.85 \mathrm{~m}$ de largo $\times 1.45 \mathrm{~m}$ de altura), al cual se le añadieron paredes de placas de unicel de $2 \mathrm{~cm}$ de grosor, forradas con papel aluminio, con la finalidad de tener un máximo de reflexión de la luz en su interior.

\section{Tratamientos con diferentes proporciones de luz LED roja y azul}

Las lámparas que se utilizaron fueron hechas a mano y estuvieron compuestas por perfiles de aluminio $(5 \mathrm{~cm}$ de ancho $\times 85 \mathrm{~cm}$ de largo) que sirvieron como base de anclaje las lámparas LED y las lámparas fluorescentes; que se colocaron en la base superior de cada uno de los gabinetes de crecimiento. Se utilizaron dos perfiles por tratamiento (12 lámparas LED por perfil), excepto para el testigo, donde se usaron cuatro perfiles (1 lámpara fluorescente por perfil) (Tabla 1).

Los tratamientos de luz que se utilizaron consistieron en diferentes proporciones de luz azul y roja y un testigo con luz fluorescente. Se utilizaron para el tratamiento A0 \%: A100 \% (A0) 24 lámparas rojos, A34.5 \%:R65.5\% (A34.5) 6 azules y 18 lámparas rojos, A57.1\%:R45.9 \% (A57.1) 12 lámparas azules y 12 lámparas rojos, A76 \%:R24\% (A76) 18 lámparas azules y 6 rojos, A100 \%:R0 \% (A100) 24 lámparas azules. La densidad de flujo de fotones fotosintéticos (DFFF) $\left(\mu \mathrm{mol} \mathrm{m}^{2}\right.$ $\mathrm{s}^{-1}$ ) y las propiedades del espectro electromagnético de los tratamientos se midió con un espectroradiómetro (Apogee instruments ${ }^{\circledast}$ modelo SS-110, EUA) y se tomaron 330 mediciones en el área de cultivo $\left(66 \mathrm{~cm} \times 20 \mathrm{~cm}=1320 \mathrm{~cm}^{2}\right)$ para verificar que los tratamientos tuvieran la misma densidad de flujo de fotones fotosintéticos (DFFF) (Tabla 1).

Se realizó un análisis estadístico de las variables físicas de los tratamientos para asegurar que las intensidades de luz entre tratamientos LED fueran iguales y las proporciones de luz azul y roja fueran diferentes; además de verificar el pico de longitud de onda (nm) e intervalo de la luz LED presente en los tratamientos. Se estableció un fotoperiodo para todos los tratamientos de luz de $15 \mathrm{~h}$ por día durante 35 días. 
Tabla 1. Propiedades físicas de los tratamientos con diferentes proporciones de luz LED azul y roja en plántula de chile habanero cultivado bajo condiciones controladas.

Table 1. Physical properties of treatments with different ratios of blue and red LED light on habanero pepper seedling grown under controlled conditions.

\begin{tabular}{|c|c|c|c|c|c|c|}
\hline & \multicolumn{6}{|c|}{ Tratamientos } \\
\hline & $\mathbf{F L}$ & AO & A34.5 & A57.1 & A76.0 & A100 \\
\hline PrA & Testigo & AO $\%$ & A34.5\% & A57.1 \% & A76 \% & $\mathrm{A} 100 \%$ \\
\hline PrR & Testigo & R100 \% & R65.5 \% & R $42.9 \%$ & R24 \% & Ro \% \\
\hline Dtot & $\begin{array}{c}119.1 \pm 0.6 \\
b^{x}\end{array}$ & $\begin{array}{c}241.5 \pm 3.1 \\
a\end{array}$ & $\begin{array}{c}241.4 \pm 3.3 \\
a\end{array}$ & $\begin{array}{c}244.4 \pm 2.4 \\
a\end{array}$ & $\begin{array}{c}241.4 \pm 1.8 \\
a\end{array}$ & $\begin{array}{c}243.8 \pm 2.5 \\
\mathrm{a}\end{array}$ \\
\hline LID & $6.4 \pm 0.0 \mathrm{~b}$ & $\begin{array}{c}13.0 \pm 0.2 \\
a\end{array}$ & $\begin{array}{c}13.0 \pm 0.2 \\
a\end{array}$ & $\begin{array}{c}13.2 \pm 0.1 \\
a\end{array}$ & $\begin{array}{c}13.0 \pm 0.1 \\
a\end{array}$ & $\begin{array}{c}13.2 \pm 0.1 \\
\mathrm{a}\end{array}$ \\
\hline$\lambda A$ & - & - & $457 \pm 0 \mathrm{~b}$ & $457 \pm 0 \mathrm{a}$ & $457 \pm 0 \mathrm{~b}$ & $457 \pm 0 \mathrm{~b}$ \\
\hline$\% \mathrm{DA}$ & - & - & $\begin{array}{c}34.5 \pm 0.6 \\
d\end{array}$ & $\begin{array}{c}57.1 \pm 0.3 \\
c\end{array}$ & $\begin{array}{c}76.0 \pm 0.3 \\
b\end{array}$ & $\begin{array}{c}100.0 \pm 0.0 \\
\mathrm{a}\end{array}$ \\
\hline$\lambda R$ & - & $636 \pm 0 \mathrm{~b}$ & $635 \pm 0 \mathrm{c}$ & $637 \pm 0$ a & $636 \pm 0 \mathrm{~b}$ & - \\
\hline$\% \mathrm{DR}$ & - & $\begin{array}{c}100.0 \pm 0.0 \\
\mathrm{a}\end{array}$ & $\begin{array}{c}65.5 \pm 0.6 \\
b\end{array}$ & $\begin{array}{c}42.9 \pm 0.3 \\
\text { c }\end{array}$ & $\begin{array}{c}24.0 \pm 0.3 \\
d\end{array}$ & - \\
\hline
\end{tabular}

${ }^{\times}$Medias con letras iguales en las filas son estadísticamente iguales (Tukey, $\mathrm{P}$ $\leq 0.05$ ). $F L=$ fluorescente con un intervalo de $416 \mathrm{~nm}-715 \mathrm{~nm}$ (testigo); $\mathrm{A}=$ Luz LED azul; R= Luz LED roja; PrA= Proporción luz azul; PrR= Proporción luz roja; Dtot= Densidad de flujo de fotones fotosintéticos total de tratamiento $\left(\mu \mathrm{mol} \mathrm{m} \mathrm{m}^{-2} \mathrm{~s}^{-1}\right) ; \mathrm{LID}=$ Luz integral diaria $\left(\mathrm{mol} \mathrm{m}^{-2} \mathrm{~d}^{-1}\right) ; \lambda \mathrm{A}=$ Pico de longitud de onda $(\mathrm{nm})$ color azul; \%DA= Porcentaje de densidad de flujo de fotones fotosintéticos de luz azul (\%); $\lambda \mathrm{R}=$ Pico de longitud de onda $(\mathrm{nm})$ color rojo; $\% \mathrm{DR}=$ Porcentaje de densidad de flujo de fotones fotosintéticos de luz roja (\%).

\section{Condiciones de crecimiento en la cámara de crecimiento}

Diecisiete días después de la siembra, las charolas con las plántulas se colocaron (una charola por tratamiento) dentro de los gabinetes de crecimiento. Las charolas se regaron diariamente con $300 \mathrm{~mL}$ de la solución Hoagland modificada a 1/5 de su concentración (Bojórquez-Quintal et al., 2014)

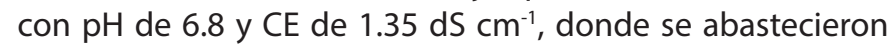
los macronutrimentos de la forma siguiente: $1.2 \mathrm{mM} \mathrm{KNO}_{3^{\prime}}$ $0.8 \mathrm{mM} \mathrm{Ca}\left(\mathrm{NO}_{3}\right)_{2}, 0.2 \mathrm{mM} \mathrm{KH}_{2} \mathrm{PO}_{4}, 0.2 \mathrm{mM} \mathrm{MgSO}_{4}$ y los micronutrimentos se abastecieron con la mezcla Tradecorp ${ }^{\circledR} \mathrm{AZ}$ $\left(0.065 \mathrm{~g} \mathrm{~L}^{-1}\right)$.

La temperatura ambiental promedio (día 28.2 \pm 0.1 ${ }^{\circ} \mathrm{C} /$ noche $25.3 \pm 0.1{ }^{\circ} \mathrm{C}$ ) y la humedad relativa promedio (día $56.2 \pm 0.5 \%$ /noche $77.1 \pm 0.5 \%$ ) se registraron cada minuto con el promedio de los registros de tres datalogger (Extech instruments ${ }^{\circledR}$ modelo RH10, USA), ubicados a nivel de copa de la planta, dentro de tres gabinetes seleccionados. La temperatura promedio de la solución nutritiva se midió con un termómetro infrarrojo (Extech instruments ${ }^{\circledR}$ modelo 42530, USA) y fue de $26.1 \pm 0.2^{\circ} \mathrm{C}$. La concentración promedio de $\mathrm{CO}_{2}$ se midió con un medidor portátil de $\mathrm{CO}_{2}$ ambiental (Extech instruments ${ }^{\circledR}$ modelo CO250, USA) y fue de $397 \pm 53$ ppm.

\section{Variables de crecimiento de la parte aérea}

Después de 35 días con los tratamientos de luz LED, las plántulas se cosecharon y se determinó de la parte aérea: la altura de planta (cm), número de hojas por planta, grosor de tallo $(\mathrm{mm})$ con un vernier digital $\left(\right.$ Truper $\left.^{\circledR}, \mathrm{MEX}\right)$, área foliar $\left(\mathrm{cm}^{2}\right.$ por planta) con un integrador de área foliar LI-3100 $\left(\right.$ LICOR $^{\circledR}$, USA), se calculó el índice de área foliar [área foliar $\left(\mathrm{cm}^{2}\right) / a ́ r e a ~ d e ~ c u l t i v o\left(\mathrm{~cm}^{2}\right)$ ], el área foliar específica [área foliar $\left(\mathrm{cm}^{2}\right) /$ peso seco hoja $\left.(\mathrm{g})\right]$, distancia de entrenudos $(\mathrm{cm})$ que se obtuvo del cociente de la altura de planta y el número de hojas.

Además, se determinó el peso fresco de tallo (g por planta) y peso fresco de hoja (g por planta) con una balanza de precisión (Ohaus ${ }^{\circledR}$ Scout Pro, USA), el peso fresco de la parte aérea (g por planta) se obtuvo la suma del peso fresco de tallo y hoja.

Posteriormente, la parte aérea se secó en una estufa de aire forzado (BINDER ${ }^{\circledR}$ modelo FED 115, DEU) por 48 horas a $70^{\circ} \mathrm{C}$ y se registró el peso seco de tallo (g por planta) y peso seco de hoja ( $\mathrm{g}$ por planta) con una balanza de precisión (Ohaus $^{\circledR}$ Golden series, USA). El peso seco de la parte aérea (g planta ${ }^{-1}$ ) se obtuvo de la suma del peso seco del tallo y de la hoja.

La frecuencia estomática (número de estomas $\mathrm{mm}^{2}$ ) se obtuvo aplicando barniz transparente de uñas en el envés de la hoja, para posteriormente despegar esta calca y contabilizar la cantidad de estomas por campo del microscopio (Carl Zeiss ${ }^{\circledR}$ Axiostar, DEU) con un aumento de 40X. En cada una de las variables medidas se utilizaron 20 repeticiones por tratamiento, con excepción de la frecuencia estomática donde se utilizaron 10 repeticiones.

\section{Concentración de pigmentos fotosintéticos en hoja}

Las concentraciones de clorofila $\mathrm{a}$, b y total (mg g ${ }^{-1}$ de peso fresco), proporción de clorofila $a / b$ y concentración de carotenoides ( $\mathrm{mg} \mathrm{g}^{-1}$ de peso fresco) se determinaron mediante la metodología descrita por la AOAC (1980). Estas variables se determinaron en hojas fuente (de reciente maduración), completamente desarrolladas, recién cortadas y utilizando tres hojas por plántula, se utilizaron nueve repeticiones por tratamiento, donde cada repetición fue una plántula.

\section{Diseño experimental y análisis estadístico}

El experimento se estableció en un diseño completamente al azar, una repetición estuvo constituida por una plántula. Los datos se sometieron a un análisis de varianza (ANOVA) y prueba de comparación de medias de Tukey $(\mathrm{P}<$ 0.05) mediante el programa SAS V9.0 (SAS Institute, 2002).

\section{RESULTADOS Y DISCUSIÓN \\ Variables de crecimiento de la parte aérea}

Los resultados de las variables de crecimiento se muestran en la Tabla 2. Las plántulas del tratamiento que recibieron $100 \%$ de luz azul, presentaron la mayor altura de plántula y distancia de entrenudos respecto al resto de tratamientos luz LED y al testigo; por otra parte, la presencia de diferentes porcentajes de luz azul y roja al mismo tiempo en los tratamientos evaluados (A34.5, A57.1 y A76.0) junto 
Tabla 2. Variables de crecimiento evaluadas en plántula de chile habanero cultivado bajo condiciones controladas con diferentes proporciones de luz LED azul y roja.

Table 2. Growth variables evaluated in habanero pepper seedling grown under controlled conditions with different ratios of blue and red LED light.

\begin{tabular}{|c|c|c|c|c|c|c|}
\hline \multicolumn{7}{|c|}{ Tratamientos } \\
\hline Var & FL & AO & A34.5 & A57.1 & A76.0 & A100 \\
\hline AP & $\begin{array}{c}7.05 \\
\pm 0.29 \mathrm{c}^{\mathrm{x}}\end{array}$ & $\begin{array}{c}9.94 \\
\pm 0.34 \mathrm{~b}\end{array}$ & $\begin{array}{c}6.81 \\
\pm 0.15 \mathrm{~cd}\end{array}$ & $\begin{array}{c}6.48 \\
\pm 0.15 \mathrm{~cd}\end{array}$ & $\begin{array}{c}5.97 \\
\pm 0.21 \mathrm{~d}\end{array}$ & $\begin{array}{c}13.55 \\
\pm 0.30 \mathrm{a}\end{array}$ \\
\hline DE & $\begin{array}{c}0.70 \\
\pm 0.04 \mathrm{c}\end{array}$ & $\begin{array}{c}1.10 \\
\pm 0.04 \mathrm{~b}\end{array}$ & $\begin{array}{c}0.70 \\
\pm 0.02 \mathrm{c}\end{array}$ & $\begin{array}{c}0.65 \\
\pm 0.02 c\end{array}$ & $\begin{array}{c}0.59 \\
\pm 0.02 c\end{array}$ & $\begin{array}{c}1.45 \\
\pm 0.04 \text { a }\end{array}$ \\
\hline DT & $\begin{array}{c}0.24 \\
\pm 0.01 \mathrm{c}\end{array}$ & $\begin{array}{c}0.29 \\
\pm 0.01 \mathrm{a}\end{array}$ & $\begin{array}{c}0.27 \\
\pm 0.01 \mathrm{ab}\end{array}$ & $\begin{array}{c}0.29 \\
\pm 0.01 \mathrm{ab}\end{array}$ & $\begin{array}{c}0.28 \\
\pm 0.01 \mathrm{ab}\end{array}$ & $\begin{array}{c}0.27 \\
\pm 0.00 \mathrm{~b}\end{array}$ \\
\hline $\mathrm{NH}$ & $\begin{array}{c}10.4 \\
\pm 0.37 \mathrm{a}\end{array}$ & $\begin{array}{c}9.25 \\
\pm 0.25 \mathrm{~b}\end{array}$ & $\begin{array}{c}9.80 \\
\pm 0.21 \mathrm{ab}\end{array}$ & $\begin{array}{c}10.1 \\
\pm 0.23 \mathrm{ab}\end{array}$ & $\begin{array}{c}10.15 \\
\pm 0.23 \mathrm{ab}\end{array}$ & $\begin{array}{c}9.45 \\
\pm 0.23 \mathrm{ab}\end{array}$ \\
\hline $\mathrm{AF}$ & $\begin{array}{c}85.05 \\
\pm 4.59 \mathrm{a}\end{array}$ & $\begin{array}{c}68.78 \\
\pm 3.11 \mathrm{~b}\end{array}$ & $\begin{array}{c}58.29 \\
\pm 2.45 \text { bc }\end{array}$ & $\begin{array}{c}50.16 \\
\pm 1.48 \mathrm{c}\end{array}$ & $\begin{array}{c}61.33 \\
\pm 1.54 \mathrm{bc}\end{array}$ & $\begin{array}{c}60.24 \\
\pm 2.74 \mathrm{bc}\end{array}$ \\
\hline IAF & $\begin{array}{c}5.15 \\
\pm 0.28 \mathrm{a}\end{array}$ & $\begin{array}{c}4.17 \\
\pm 0.19 \mathrm{~b}\end{array}$ & $\begin{array}{c}3.54 \\
\pm 0.15 \mathrm{bc}\end{array}$ & $\begin{array}{c}3.04 \\
\pm 0.09 c\end{array}$ & $\begin{array}{c}3.72 \\
\pm 0.09 \mathrm{bc}\end{array}$ & $\begin{array}{c}3.64 \\
\pm 0.17 \mathrm{bc}\end{array}$ \\
\hline AFE & $\begin{array}{l}566.50 \\
\pm 15.5 \mathrm{a}\end{array}$ & $\begin{array}{c}452.71 \\
\pm 18.51 \mathrm{~b}\end{array}$ & $\begin{array}{c}376.14 \\
\pm 19.0 \mathrm{~cd}\end{array}$ & $\begin{array}{l}325.70 \\
\pm 12.4 \mathrm{~d}\end{array}$ & $\begin{array}{c}415.48 \\
\pm 16.9 \mathrm{bc}\end{array}$ & $\begin{array}{l}449.51 \\
\pm 10.2 \mathrm{~b}\end{array}$ \\
\hline $\mathrm{FE}$ & $\begin{array}{c}86.60 \\
\pm 4.28 \mathrm{~d}\end{array}$ & $\begin{array}{r}153.60 \\
\pm 6.09 \mathrm{c}\end{array}$ & $\begin{array}{r}239.60 \\
\pm 5.34 \mathrm{a}\end{array}$ & $\begin{array}{r}189.90 \\
\pm 6.20 \mathrm{~b}\end{array}$ & $\begin{array}{r}149.80 \\
\pm 4.07 \mathrm{c}\end{array}$ & $\begin{array}{c}165.40 \\
\pm 8.34 \mathrm{bc}\end{array}$ \\
\hline
\end{tabular}

${ }^{\times}$Medias $\pm \mathrm{DE}$ con igual letra en las filas son estadísticamente iguales (Tukey, $\mathrm{P} \leq$ 0.05). $\mathrm{ns}=$ No significativo; $\mathrm{Var}=$ Variables; $\mathrm{FL}=$ fluorescente (testigo); $\mathrm{AP}=$ Altura de planta $(\mathrm{cm}) ; \mathrm{DE}=$ Distancia entrenudos $(\mathrm{cm}) ; \mathrm{DT}=$ Diámetro de tallo $(\mathrm{cm}) ; \mathrm{NH}=$ Número de hojas; $\mathrm{AF}=$ Área foliar $\left(\mathrm{cm}^{2}\right.$ por planta); $\mathrm{IAF}=$ Índice de área foliar; $\mathrm{AFE}=$ Área foliar específica $\left(\mathrm{cm}^{2} \mathrm{~g}^{-1}\right) ; \mathrm{FE}=$ Frecuencia estomática (número de estomas $\mathrm{mm}^{2}$ ).

con el testigo, generó las menores alturas y distancia de entrenudos en plántula en comparación con los tratamientos monocromáticos rojo (A0) y azul (A100) (Tabla 2). El diámetro de tallo fue mayor en plantas que recibieron tratamientos con luz LED respecto al testigo, y las plantas que tuvieron $0 \%$ de luz azul generó un mayor diámetro de tallo respecto a las plantas que recibieron $100 \%$ de luz azul (Tabla 2). Aunque no hubo efectos significativos en el número de hojas entre los tratamientos de luz LED, las plantas del testigo resultaron con un número de hojas mayor que las plantas que recibieron 0 $\%$ de luz azul (Tabla 2). Las plantas del testigo obtuvieron un área foliar, índice de área foliar y área foliar específica mayor en comparación con los diversos tratamientos con luz LED, además las plantas del tratamiento A57.1 tuvieron una menor área foliar e índice de área foliar en relación con las que recibieron $0 \%$ de luz azul; de manera similar el área foliar específica fue menor en las plantas del tratamiento A57.1 en comparación con las que recibieron $0 \%$ y $100 \%$ de luz azul (Tabla 2). La frecuencia estomática fue mayor en plantas que recibieron un $34.5 \%$ de luz azul en comparación con las plantas del testigo y con el resto de tratamientos con luz LED (Tabla 2).

Verma et al. (2018) encontraron en plantas de Digitalis purpurea resultados parecidos a los del presente estudio respecto a la altura de planta en donde nosotros usamos tratamientos similares a los de ellos, mientras que lo que registraron Hernández y Kubota (2016) en plántula de pepino, difiere con lo que se obtuvo en chile habanero, ya que sus resultados se ajustaron a una regresión lineal en donde a mayor porcentaje de luz azul respecto a luz roja la altura de plántula disminuyó, mientras que en plántula de tomate, Kim y Hwang (2019) no encontraron efectos en altura de plántula al usar $0 \%, 30 \%, 50 \%, 70 \%, 100 \%$ de luz azul respecto a luz roja y un testigo de luz fluorescente.

Las menores alturas de plántula y distancia de entrenudos que se registraron en los tratamientos A34.5, A57.1 y A76.0, pudo deberse a la existencia de un efecto negativo que tuvieron las plántulas que recibieron luz azul y roja al mismo tiempo, en comparación con los tratamientos monocromáticos A0 y A100 (Tabla 2). El efecto negativo pudo darse entre los fotorreceptores fitocromo (asociado a luz roja) y los criptocromos, fototropinas y zeitlupe (asociados a luz azul), ya que al formar parte del sistema de fotorregulación de señalización intercelular con segundos mensajeros, pudo haber alterado el transporte, activación y síntesis hormonal; además de la activación y expresión de genes, entre otros procesos, ya que los fotorreceptores regulan y contribuyen en diversos procesos fisiológicos de manera redundante o sinérgica (Berkovich et al., 2017; Demotes-Mainard et al., 2016).

Las plantas que recibieron solo $100 \%$ de luz azul, tuvieron un comportamiento opuesto a lo asociado a la luz azul, la cual tiende a reducir la altura de planta a través del control sobre la expansión celular y la reducción de la extensibilidad de la pared celular (Huché-Thélier et al., 2016); dado que, por el contrario, la luz roja promueve una estimulación activa del fotorreceptor fitocromo el cual está asociado en promover una mayor altura de planta mediante la elongación del tallo (Pocock, 2015) y por consiguiente la distancia de entrenudos. El efecto asociado a la luz roja en las plántulas de habanero se observó de manera parcial, ya que las que recibieron solo luz roja (A0) tuvieron una mayor altura de plántula y distancia de entrenudos que las que recibieron luz azul y roja al mismo tiempo junto con el tratamiento fluorescente, pero con menores valores que las que recibieron $100 \%$ de luz azul (Tabla 2).

La baja altura de plántula y distancia de entrenudos que se encontró en el tratamiento fluorescente en comparación con las plantas que recibieron $0 \%$ y $100 \%$ de luz azul pudo deberse a que el testigo tuvo la mitad de la LID (luz integral diaria) que el resto de los tratamientos LED (Tabla 1).

Kim y Hwang (2019) y Hernández et al. (2016) encontraron en plántulas de tomate resultados diferentes en el diámetro de tallo en comparación con los de chile habanero; respecto a los resultados de Kim y Hwang (2019), los tratamientos con diferentes porcentajes de luz azul y roja junto con un testigo de luz fluorescente no tuvieron efectos, mientras que lo que registraron Hernández et al. (2016) fue que $50 \%$ de luz azul respecto a luz roja presentó el mayor diámetro de tallo en comparación con las que recibieron 0 $\%$ y $100 \%$ de luz azul que fueron las que menor diámetro de tallo presentaron.

De una manera similar que se describió en altura de plántula y distancia de entrenudos, el diámetro de tallo fue 
menor cuando recibieron luz fluorescente lo más probable que debido a que el testigo tuvo la mitad de la LID (Tabla 1) que el resto de los tratamientos LED, trajo consigo una disminución del diámetro de tallo respecto al resto de tratamientos con luz LED.

Las plántulas del testigo fluorescente presentaron de manera general un efecto directo y constante en el crecimiento bajo este tratamiento, debido a que la LID (luz integral diaria) necesaria para la producción de plántula en ambiente controlado es de $13 \mathrm{~mol} \mathrm{~m}^{-2} \mathrm{~d}^{-1}$ (Kubota, 2016), sin embargo el testigo fluorescente solo aportó $\left(6.4 \mathrm{~mol} \mathrm{~m}^{-2}\right.$ $\mathrm{d}^{-1}$ ) (Tabla 1), lo cual es inferior y por debajo de la mitad de lo necesario, mientras que en los tratamientos con luz LED tuvieron una LID entre 13.0 y $13.2 \mathrm{~m}^{-2} \mathrm{~d}^{-1}$ (Tabla 1 ), los cuales se encuentran dentro del valor necesario para la producción de plántula según lo mencionado por Kubota (2016) que es de $13 \mathrm{~mol} \mathrm{~m}^{2} \mathrm{~d}^{-1}$.

A causa de lo anterior, las plantas del tratamiento testigo presentaron una adaptación a baja intensidad luminosa y las características que poseen las plantas con esta adaptación, típicamente tienen una baja tasa fotosintética, pero poseen mayor eficiencia de captura y uso de la energía de la luz, usualmente a través del incremento en la concentración de clorofila y una disminución de la relación clorofila $\mathrm{a} / \mathrm{b}$, mientras que las plantas adaptadas a ambientes con mayor intensidad luminosa poseen puntos de saturación de luz más altos respecto a la tasa fotosintética neta, debido a que poseen en el proceso de la fotosíntesis una cadena de transporte de electrones más eficiente y una mayor concentración de enzimas para la carboxilación (Qiansheng et al., 2014), además de que se lleva a desarrollar hojas más grandes de menor grosor y baja frecuencia estomática (Stanton et al., 2010), con el objetivo de tener un área foliar suficiente para que se pueda llevar a cabo de la mejor manera el proceso fotosíntesis, fijación de $\mathrm{CO}_{2}$ y acumulación de almidón (Tsukaya, 2005). Estas características de adaptación también se observaron en el experimento relacionado a los resultados de las variables evaluadas asociadas con el órgano de la hoja, como es el caso de número de hojas, área foliar, índice de área foliar, área foliar específica, frecuencia estomática y peso fresco de hoja (Tabla 2 y 3 ).

Hernández y Kubota (2016) encontraron en plántulas de pepino resultados similares en el número de hojas, ya que no existieron resultados estadísticamente significativos cuando se utilizó tratamientos con diferentes proporciones de luz azul y roja. Por otro lado, en plantas de Digitalis purpurea Verma et al. (2018) hallaron que cuando estas recibieron $20 \%$ de luz azul respecto a luz roja tuvieron mayor número de hojas que las que recibieron $0 \%, 50 \%, 80 \%$ y $100 \%$ de luz azul junto con las del testigo de luz fluorescente. De manera diferente a los resultados del presente estudio en chile habanero, en plántulas de tomate Kim y Hwang (2019) encontraron que las que tuvieron $30 \%$ de luz azul presentaron mayor número de hojas en comparación con las del testigo fluorescente y con las que tuvieron $50 \%, 70 \%$ y $100 \%$ de luz azul respecto a luz roja.

Tabla 3. Peso fresco y seco evaluado en plántula de chile habanero cultivado bajo condiciones controladas con diferentes proporciones de luz LED azul y roja.

Table 3. Fresh and dry weight evaluated in habanero pepper seedling, grown under controlled conditions with different ratios of blue and red LED light.

\begin{tabular}{|c|c|c|c|c|c|c|}
\hline \multicolumn{7}{|c|}{ Tratamientos } \\
\hline Var & $\mathbf{F L}$ & AO & A34.5 & A57.1 & A76.0 & A100 \\
\hline PFT & $\begin{array}{c}0.26 \\
\pm 0.02 \mathrm{c}\end{array}$ & $\begin{array}{c}0.52 \\
\pm 0.03 \mathrm{~b}\end{array}$ & $\begin{array}{c}0.29 \\
\pm 0.02 c\end{array}$ & $\begin{array}{c}0.32 \\
\pm 0.02 \mathrm{c}\end{array}$ & $\begin{array}{c}0.32 \\
\pm 0.02 \mathrm{c}\end{array}$ & $\begin{array}{c}0.62 \\
\pm 0.02 \mathrm{a}\end{array}$ \\
\hline PFH & $\begin{array}{c}1.62 \\
\pm 0.08 \mathrm{a}\end{array}$ & $\begin{array}{c}1.40 \\
\pm 0.07 \mathrm{ab}\end{array}$ & $\begin{array}{c}1.34 \\
\pm 0.05 \mathrm{~b}\end{array}$ & $\begin{array}{c}1.23 \\
\pm 0.04 \mathrm{~b}\end{array}$ & $\begin{array}{c}1.45 \\
\pm 0.04 \mathrm{ab}\end{array}$ & $\begin{array}{c}1.30 \\
\pm 0.05 \mathrm{~b}\end{array}$ \\
\hline PFA & $\begin{array}{c}1.92 \\
\pm 0.09 \text { a }\end{array}$ & $\begin{array}{c}1.92 \\
\pm 0.08 \mathrm{a}\end{array}$ & $\begin{array}{c}1.63 \\
\pm 0.05 \mathrm{bc}\end{array}$ & $\begin{array}{c}1.56 \\
\pm 0.05 c\end{array}$ & $\begin{array}{c}1.78 \\
\pm 0.05 \mathrm{abc}\end{array}$ & $\begin{array}{c}1.88 \\
\pm 0.07 \mathrm{a}\end{array}$ \\
\hline PST & $\begin{array}{c}0.03 \\
\pm 0.00 \mathrm{~d}\end{array}$ & $\begin{array}{c}0.08 \\
\pm 0.01 \mathrm{ab}\end{array}$ & $\begin{array}{c}0.06 \\
\pm 0.01 \mathrm{bc}\end{array}$ & $\begin{array}{c}0.06 \\
\pm 0.00 \mathrm{c}\end{array}$ & $\begin{array}{c}0.05 \\
\pm 0.01 \mathrm{~cd}\end{array}$ & $\begin{array}{c}0.09 \\
\pm 0.00 \mathrm{a}\end{array}$ \\
\hline PSH & $\begin{array}{c}0.15 \\
\pm 0.01 \mathrm{~ns}\end{array}$ & $\begin{array}{c}0.16 \\
\pm 0.01 \mathrm{~ns}\end{array}$ & $\begin{array}{c}0.16 \\
\pm 0.01 \mathrm{~ns}\end{array}$ & $\begin{array}{c}0.16 \\
\pm 0.01 \mathrm{~ns}\end{array}$ & $\begin{array}{c}0.15 \\
\pm 0.01 \mathrm{~ns}\end{array}$ & $\begin{array}{c}0.13 \\
\pm 0.01 \mathrm{~ns}\end{array}$ \\
\hline PSA & $\begin{array}{c}0.19 \\
\pm 0.01 \mathrm{~ns}\end{array}$ & $\begin{array}{c}0.23 \\
\pm 0.01 \mathrm{~ns}\end{array}$ & $\begin{array}{c}0.22 \\
\pm 0.01 \mathrm{~ns}\end{array}$ & $\begin{array}{c}0.21 \\
\pm 0.01 \mathrm{~ns}\end{array}$ & $\begin{array}{c}0.20 \\
\pm 0.01 \mathrm{~ns}\end{array}$ & $\begin{array}{c}0.23 \\
\pm 0.01 \mathrm{~ns}\end{array}$ \\
\hline
\end{tabular}

${ }^{\times}$Medias $\pm \mathrm{DE}$ con igual letra en las filas son estadísticamente iguales (Tukey, $\mathrm{P} \leq$ 0.05). $\mathrm{ns}=$ No significativo; $\mathrm{Var}=$ Variables; $\mathrm{FL}=$ fluorescente (testigo); $\mathrm{PFT}=$ Peso fresco de tallo ( $\mathrm{g}$ por planta); $\mathrm{PFH}=$ Peso fresco de hoja ( $\mathrm{g}$ por planta); $\mathrm{PFA}=$ Peso fresco de la parte aérea ( $g$ por planta); $\mathrm{PST}=$ Peso seco de tallo (g por planta); $\mathrm{PSH}=$ Peso seco de hoja ( $g$ por planta); $\mathrm{PSA}=$ Peso seco de la parte aérea (g por planta).

El número de hojas se promovió en mayor proporción en plantas que recibieron luz fluorescente, a pesar de que tuvieron la mitad de la LID (Tabla 1); estas tuvieron estadísticamente el mismo número de hojas que los tratamientos con luz LED e inclusive tuvieron mayor cantidad de hojas que el tratamiento A0, lo anterior pudo deberse a causa de un mecanismo de adaptación a baja intensidad luminosa por parte de la planta (Qiansheng et al., 2014; Stanton et al., 2010; Tsukaya, 2005). Entre los tratamientos con luz LED, esta especie no es tan susceptible a modificar su número de hojas con base en una cierta proporción de luz azul o roja.

En plántulas de tomate se encontró, por parte de Kim y Hwang (2019), un comportamiento diferente en el área foliar, ya que las que recibieron $0 \%$ y $100 \%$ de luz azul respecto a luz roja tuvieron menor área foliar que las que recibieron 30 $\%$ y $70 \%$ de luz azul al igual que las de un tratamiento testigo fluorescente. De manera similar a los anteriores autores, Hernández et al. (2016) registraron en plántulas de tomate que tanto las que recibieron $0 \%$ y $100 \%$ de luz azul respecto a roja tuvieron la menor área foliar en comparación con las que tuvieron $10 \%, 30 \%, 50 \%, 75 \%$ de luz azul y también con el testigo fluorescente. Por otro lado, los resultados encontrados en área foliar de plántulas de pepino por Hernández y Kubota (2016) se ajustaron a una regresión lineal en donde a mayor porcentaje de luz azul respecto a luz roja el área foliar disminuyó.

La mayor área foliar que se presentó en las plantas que crecieron bajo luz fluorescente (Tabla 2), esto pudo deberse a un mecanismo de adaptación de las plántulas a un entorno de baja intensidad luminosa (Qiansheng et al., 2014) que se presentó con la luz fluorescente lo que condujo un mayor de- 
sarrollo en el tamaño de las hojas (Stanton et al., 2010), para así obtener área foliar suficiente para poder llevar a cabo de manera correcta el proceso de la fotosíntesis (Tsukaya, 2005).

El comportamiento entre los tratamientos de luz LED evaluados en plántulas de chile habanero, pudo ser inherente al tipo de luz y la especie, ya que la luz azul puede incrementar o disminuir el área foliar y en algunas especies no tiene ningún efecto (Huché-Thélier et al., 2016), mientras la luz roja puede incrementar o disminuir el área foliar dependiendo de la biología de la planta (Berkovich et al., 2017).

El índice de área foliar, en plántulas con tratamientos de luz LED, estuvieron dentro del intervalo de 3.0 a 4.0 (Tabla 2), con lo cual existió una intercepción de luz de un $90 \%$ a 100 $\%$, mientras que en el testigo al haber sido $<4.0$ (Tabla 2), las hojas más bajas de la planta se encontraron muy sombreadas y no recibieron suficiente luz incidente, lo que pudo haber generado una pérdida de fotosintatos a través de una mayor respiración (Yamori, 2016). El comportamiento del índice de área foliar en plántulas con el tratamiento fluorescente, fue el mismo que se presentó en la variable de área foliar, en donde debido a una adaptación a una baja intensidad luminosa (Qiansheng et al., 2014) que se presentó con las plántulas del tratamiento fluorescente, se ocasionó a desarrollar hojas más grandes (Stanton et al., 2010), con el objetivo de tener un área foliar suficiente para que se pueda llevar a cabo de la mejor manera el proceso fotosíntesis (Tsukaya, 2005).

Agarwal et al. (2018) encontraron un comportamiento parecido a los del presente estudio en plantas de espinaca, en donde las que recibieron $0 \%$ y $100 \%$ de luz azul respecto a luz roja tuvieron mayor área foliar específica que las de 25 $\%, 50 \%$ y $75 \%$ de luz azul.

El área foliar específica (AFE) es una medida indirecta del grosor de la hoja en una planta (Amanullah, 2015), lo cual se traduce en que a una mayor AFE menor grosor de hoja y viceversa. El menor grosor de hoja (AFE) se obtuvo en las plantas del testigo (Tabla 2), se debió a una adaptación a una baja intensidad luminosa, que llevó a desarrollar hojas más grandes de menor grosor y baja frecuencia estomática (Stanton et al., 2010), con el objetivo de tener un área foliar suficiente para que se pueda llevar a cabo de la mejor manera el proceso fotosíntesis, fijación de $\mathrm{CO}_{2}$ y acumulación de almidón (Tsukaya, 2005).

Entre diferentes tipos de luces monocromáticas, se ha visto que la luz azul promueve más el grosor de la hoja que la luz roja, verde o combinaciones con altos porcentajes de luz azul (Huché-Thélier et al., 2016). Este comportamiento difiere a lo observado en plántulas de chile habanero con diferentes tratamientos de luz azul y roja LED, lo cual pudo deberse a un proceso de aclimatación inherente a la especie al espectro de luz incidente (Berkovich et al., 2017).

Shengxin et al. (2016) encontraron en plántulas de colza que diferentes tratamientos con luz azul y roja LED no tuvieron ningún efecto en la frecuencia estomática. Por otra parte, en plantas de Digitalis pupurea que recibieron porcentajes de $0 \%, 20 \%, 50 \%, 80 \%$ y $100 \%$ de luz azul respecto a luz roja los resultados de la frecuencia estomática fueron estadísticamente iguales entre estos, sin embargo, las plantas del testigo fluorescente tuvieron mayor frecuencia estomática que las que recibieron $50 \%$ de luz azul (Verma et al., 2018).

Las plantas adaptadas a una baja intensidad luminosa tienden a tener una baja frecuencia estomática (Stanton et al., 2010), lo cual sucedió en las plántulas del testigo fluorescente al tener la mitad de la LID respecto a los tratamientos con luz LED (Tabla 1). Por otro lado, se ha observado que la luz monocromática azul tiende a incrementar la frecuencia estomática en comparación con luz monocromática roja o verde (Huché-Thélier et al., 2016), sin embargo, en chile habanero se puede concluir que un $34.5 \%$ de luz azul indujo la mayor frecuencia estomática.

Los resultados de las variables de peso fresco evaluado en plántula se muestran en el Tabla 3. El peso fresco de tallo fue mayor en plantas que tuvieron el $100 \%$ de luz azul respecto al resto de plantas con diferentes tratamientos con luz LED y las plantas del testigo (Tabla 3). A pesar de que no existió diferencias estadísticas en el peso fresco de hoja en las plantas que recibieron los diferentes tratamientos LED, las plantas del testigo tuvieron el mayor peso fresco de hoja en comparación con las que recibieron $34.5 \%, 57.1 \%$ y $100 \%$ de luz azul (Tabla 3). El peso fresco de la parte aérea fue mayor para las plantas que recibieron $0 \%$ y $100 \%$ de luz azul, así como para el testigo en relación con las que recibieron 34.5 $\%$ y $57.1 \%$ de luz azul (Tabla 3). Las plantas que recibieron luz azul al $100 \%$ mostraron el mayor peso seco de tallo en comparación con las que recibieron $34.5 \%, 57.1 \%, 76 \%$ de luz azul y con el testigo fluorescente (Tabla 3 ).

Kim y Hwang (2019) encontraron en plántula de tomate un comportamiento diferente, ya que el mayor peso fresco de tallo se encontró en plántulas que recibieron $0 \%$ de luz azul respecto a luz roja de las que tuvieron $50 \%, 70 \%$, $100 \%$ de luz azul y un testigo fluorescente.

El menor peso fresco de tallo se registró en los tratamientos A34.5, A57.1 y A76.0, lo cual pudo deberse a la existencia de un efecto negativo que tuvieron las plántulas que recibieron luz azul y roja al mismo tiempo, en comparación con los tratamientos monocromáticos A0 y A100 (Tabla 2). Este efecto negativo podría explicarse con base a un proceso fisiológico a nivel de fotorreceptores asociados con luz azul y roja, los cuales al generar mecanismos de señalización intercelular (segundos mensajeros) al ser activados por la luz roja y/o azul, podrían estar generando una comunicación redundante entre estos, lo cual podría ocasionar que procesos de transporte, activación y síntesis hormonal, activación y expresión de genes, entre otros (Berkovich et al., 2017; Demotes-Mainard et al., 2016) se alteren y en consecuencia generen un menor crecimiento en los tejidos de las plántulas que recibieron luz azul y roja al mismo tiempo por lo cual hubo menos peso fresco de hoja que los que recibieron luz monocromática azul y roja (Tabla 3).

En plantas de Digitalis purpurea se encontró resultados diferentes respecto al peso fresco de hoja, en donde fue mayor en plantas que recibieron $20 \%$ de luz azul respecto 
a luz roja que las que recibieron $0 \%, 50 \%, 75 \%$ y $100 \%$ de luz azul, al igual que las del tratamiento testigo fluorescente (Verma et al., 2018). Por otra parte, en plántulas de tomate se encontró que plántulas que tuvieron tratamientos con $0 \%$, $30 \%$ y $70 \%$ de luz azul respecto a luz roja, tuvieron el mayor peso fresco de hoja respecto a las que recibieron $100 \%$ de luz azul (Kim y Hwang, 2019).

Las características de las plantas adaptadas a bajas intensidades de luz (Qiansheng et al., 2014), están asociadas a tener hojas más grandes (mayor área foliar) (Stanton et al., 2010) para poder llevar a cabo de la mejor manera el proceso fotosíntesis (Tsukaya, 2005), esta adaptación se presentó en las plántulas del testigo fluorescente, debido a que estas recibieron la mitad de la LID en comparación con las que recibieron luz LED (Tabla 1), lo cual generó un mayor peso fresco de hoja que los tratamientos que recibieron $34.5 \%, 57.1 \%$ y $100 \%$ de luz azul (Tabla 2). Lo anterior se pudo corroborar, ya que el efecto de la adaptación a baja intensidad de luz, se observó también en las variables de crecimiento asociadas con las hojas de plántula (Tabla 2).

Existieron tres comportamientos generales en los resultados relacionados con el peso fresco de tallo y hoja en plántulas de chile habanero (Tabla 3).

En el primer comportamiento se promovió el desarrollo del tallo en plántulas que solo recibieron luz azul (A100) y se corroboró con los resultados de altura de planta, distancia de entrenudos y peso fresco de tallo (Tabla 2 y 3 ). En el segundo comportamiento se promovió el desarrollo de las hojas en plántulas que recibieron luz fluorescente y se corroboró con el diámetro de tallo, número de hojas, área foliar, índice de área foliar, área foliar específica, frecuencia estomática y peso fresco de hoja (Tabla 2 y 3 ).

En el primer comportamiento el tallo fue un órgano de demanda con mayor prioridad de fotosintatos para su crecimiento respecto a las hojas, y en el segundo comportamiento fue la hoja el órgano de mayor prioridad respecto al tallo (Engels et al., 2012).

En el tercer comportamiento existió un efecto negativo en los resultados de las variables de altura de plántula, distancia de entrenudos y peso fresco de tallo en plántulas que recibieron al mismo tiempo luz azul y roja, en comparación con los tratamientos monocromáticos azul y rojo (Tabla 2 y 3). Esto pudo deberse a un efecto negativo a nivel de fotorreceptores, ya que estos forman parte del sistema de fotorregulación de señalización intercelular con segundos mensajeros, el cual regula la mayoría de los procesos fisiológicos de plantas (Berkovich et al., 2017).

En relación al peso fresco de la parte aérea Hernández y Kubota (2016) registraron en plántula de pepino un comportamiento diferente a los resultados del presente estudio, ya que las plantas que recibieron tratamientos con diferentes porcentajes de luz azul y roja sus resultados se ajustaron a una regresión lineal en donde a mayor porcentaje de luz azul respecto a la luz roja el peso fresco de la parte aérea disminuyó. Hernández et al. (2016) encontraron un comportamiento diferente en plántulas de tomate donde el mayor peso fresco de la parte aérea fue en las plantas que recibieron $10 \%$ y $50 \%$ de luz azul respecto a la roja y de manera contraria los menores pesos estuvieron en las plantas que recibieron el testigo fluorescente y las que tuvieron $0 \%$ y $100 \%$ de luz azul. Por otro lado, Kim y Hwang (2019) en plántulas de tomate el mayor peso fresco de la parte aérea se registró con un tratamiento $0 \%$ y $30 \%$ de luz azul respecto a luz roja y el menor peso fresco en las que recibieron $50 \%$ de luz azul. Los diferentes comportamientos de resultados de peso fresco de la parte aérea que obtuvieron los anteriores autores mencionados en cada investigación individual junto con los resultados del presente estudio a pesar de que nosotros utilizamos tratamientos de luz LED azul y roja en proporciones similares a los autores mencionados, una posible causa de este comportamiento se debió a que la composición espectral de la luz posee un efecto regulatorio fuerte en la morfogénesis de las plantas, el estado hormonal, la ontogénesis, el metabolismo secundario y el crecimiento de la planta, sin embargo, los resultados de estos efectos varían entre especies e inclusive entre cultivares (Berkovich et al., 2017).

El peso fresco de la parte aérea, al ser la suma del peso fresco de tallo más la hoja, se observó un comportamiento diferente al de la suma de sus partes individuales (Tabla 3), mientras que el tratamiento $\mathrm{A} 100$ generó que las plántulas tuvieran el mayor peso fresco de tallo y el menor peso fresco de hoja respecto a las de la luz fluorescente y por el contrario, las plántulas del tratamiento fluorescente tuvieron menor peso fresco de tallo y mayor peso de hoja que las del tratamiento A100, el peso fresco de la parte aérea fue estadísticamente el mismo en ambos tratamientos (Tabla 3). Esto corroboró que el testigo fluorescente promovió el desarrollo de la hoja en decremento del tallo y el tratamiento A100 promovió el desarrollo del tallo en decremento de la hoja, pero sin afectar al final la materia fresca total de la plántula (Tabla 3).

De manera contraria, Kim y Hwang (2019) encontraron en plántulas de tomate, que diferentes porcentajes de luz azul y roja, además de un tratamiento fluorescente, no generaron diferencias estadísticas sobre peso seco de tallo y, por el contrario, en peso seco de hoja y peso seco de la parte aérea si existieron resultados con diferencias estadísticas.

Se registraron efectos en el peso seco de hoja en plantas de Digitalis purpurea que estuvieron bajo diferentes tratamientos de luz azul y roja (Verma et al., 2018), de manera similar, en plántulas de tomate (Hernández et al., 2016) y en plántulas de pepino (Hernández y Kubota, 2016) tratamientos con diferentes proporciones de luz azul y roja tuvieron efecto en el peso seco de la parte aérea.

Se pudo observar que a pesar de que existió tres comportamientos en las variables de crecimiento que se midieron en plántulas frescas anteriormente descritas (Tabla 2 y 3), al momento de secarlas se encontró que aunque los tratamientos tuvieron efecto en todas las variables de crecimiento que se midieron, al final el peso seco de la parte área (biomasa total de la parte aérea de la plántula) fue estadísticamente igual para todos los tratamientos con luz LED como también para el testigo fluorescente (Tabla 4). 
Tabla 4. Pigmentos fotosintéticos en hojas en plántula de chile habanero cultivado bajo condiciones controladas con diferentes proporciones de luz LED azul y roja.

Table 4. Photosynthetic pigments and phenolic compounds in habanero pepper seedling leaves, grown under controlled conditions with different proportions of blue and red LED light.

\begin{tabular}{ccccccc}
\hline \multicolumn{7}{c}{ Tratamientos } \\
\hline Variables & FL & A0 & A34.5 & A57.1 & A76.0 & A100 \\
\hline \multirow{2}{*}{ Cla } & 1.12 & 0.97 & 1.04 & 0.92 & 1.07 & 0.95 \\
& $\pm 0.05 \mathrm{a}^{\times}$ & $\pm 0.04 \mathrm{ab}$ & $\pm 0.06 \mathrm{ab}$ & $\pm 0.04 \mathrm{~b}$ & $\pm 0.06 \mathrm{ab}$ & $\pm 0.03 \mathrm{ab}$ \\
$\mathrm{Clb}$ & 0.45 & 0.41 & 0.45 & 0.39 & 0.43 & 0.40 \\
& $\pm 0.10 \mathrm{~ns}$ & $\pm 0.01 \mathrm{~ns}$ & $\pm 0.09 \mathrm{~ns}$ & $\pm 0.02 \mathrm{~ns}$ & $\pm 0.10 \mathrm{~ns}$ & $\pm 0.02 \mathrm{~ns}$ \\
Cltot & 1.57 & 1.38 & 1.49 & 1.31 & 1.50 & 1.35 \\
& $\pm 0.07 \mathrm{~ns}$ & $\pm 0.05 \mathrm{~ns}$ & $\pm 0.08 \mathrm{~ns}$ & $\pm 0.06 \mathrm{~ns}$ & $\pm 0.09 \mathrm{~ns}$ & $\pm 0.05 \mathrm{~ns}$ \\
Cla/b & 1.95 & 2.36 & 2.09 & 2.34 & 2.24 & 2.42 \\
& $\pm 0.21 \mathrm{~ns}$ & $\pm 0.05 \mathrm{~ns}$ & $\pm 0.16 \mathrm{~ns}$ & $\pm 0.03 \mathrm{~ns}$ & $\pm 0.18 \mathrm{~ns}$ & $\pm 0.05 \mathrm{~ns}$ \\
Car & 4.20 & 3.80 & 4.10 & 3.70 & 4.20 & 3.70 \\
& $\pm 0.17 \mathrm{~ns}$ & $\pm 0.12 \mathrm{~ns}$ & $\pm 0.18 \mathrm{~ns}$ & $\pm 0.18 \mathrm{~ns}$ & $\pm 0.23 \mathrm{~ns}$ & $\pm 0.13 \mathrm{~ns}$ \\
\hline
\end{tabular}

'Medias con igual letra en las filas son estadísticamente iguales (Tukey, $\mathrm{P} \leq$ 0.05). $\mathrm{ns}=$ No significativo; $\mathrm{FL}=$ fluorescente (testigo); $\mathrm{Cla}=$ Clorofila a (mg $\mathrm{g}^{-1}$ de peso fresco); $\mathrm{Clb}=$ Clorofila $\mathrm{b}\left(\mathrm{mg} \mathrm{g}^{-1}\right.$ de peso fresco); Cltot= Clorofila total ( $\mathrm{mg} \mathrm{g}^{-1}$ de peso fresco); $\mathrm{Cla} / \mathrm{b}=$ Clorofila $\mathrm{a} / \mathrm{b}$; $\mathrm{Car}=$ Carotenoides ( $\mathrm{mg} \mathrm{g}^{-1}$ de peso fresco).

Este resultado se puede atribuir con base en el proceso de acumulación de biomasa que se llevó a cabo a través del crecimiento, el cual está constituido por las etapas de división y expansión celular (Cosgrove, 2005). El crecimiento está regulado por ciclos circadianos, condiciones ambientales, nutrimentales, hídricas y mediante la señalización con segundos mensajeros y fitohormonas (Poiré et al., 2010; Walter et al., 2009), siendo esta última usadas por los fotorreceptores como uno de sus mecanismos de comunicación en la planta (Berkovich et al., 2017) y además poseen un papel como moléculas reguladoras de crecimiento y desarrollo (Engels et al., 2012).

Dadas las bases fisiológicas del crecimiento, la razón por la cual no existió efectos en la biomasa total de la parte aérea de la plántula en comparación con las variables de crecimiento que se midieron con la plántula en estado fresco, se debió a la diferencia del grado de expansión celular que se presentó en el tejido del tallo y hoja dependiendo del grado de la relación fuente-demanda que ejercieron los diferentes tipos de luz evaluados en ambos tejidos, ya que la expansión celular puede llevarse a cabo en dos o tres dimensiones o por elongación a una dimensión (Carpita et al., 2015).

Al eliminar el agua intercelular de los tejidos frescos, la materia seca que permanece en la planta consiste en más del $90 \%$ como celulosa, almidón, lípidos, proteínas y está directamente relacionada con la síntesis de compuestos orgánicos a través de la fotosíntesis (Engels et al., 2012), lo que demostró que debió de existir una modificación en el volumen intercelular (expansión celular) en el tejido fresco de tallo y hoja respecto a cada tratamiento de luz que se usó, con lo cual provocó una redistribución de biomasa entre ambos tejidos, resultando en una ganancia idéntica de biomasa en la parte aérea de las plántulas sin importar el tratamiento de luz (Tabla 4).

\section{Concentración de pigmentos fotosintéticos en hoja}

Los resultados de la concentración de los pigmentos fotosintéticos evaluados en hoja de plántulas de habanero se muestran en el Tabla 4. A pesar de que no existió diferencias estadísticas en la concentración de clorofila a en hojas de plantas que recibieron los diferentes tratamientos de luz LED, las plantas del testigo tuvieron la mayor concentración respecto a las que recibieron $57.1 \%$ de luz azul (Tabla 4).

Verma et al. (2018) encontraron en plantas de Digitalis pupurea un comportamiento diferente en la concentración de clorofila a, ya que en plantas que recibieron $100 \%$ de luz azul tuvieron mayor concentración que las que recibieron 20 $\%, 50 \%$ y $80 \%$ de luz azul respecto a luz roja, sin embargo, al compararse con la que recibieron solo luz roja no tuvieron diferencias estadísticas.

El comportamiento de la concentración de clorofila a en el testigo, pudo deberse a que al tener la mitad de la luz integral diaria (LDI) $6.4\left(\mathrm{~mol} \mathrm{~m}^{-2} \mathrm{~d}^{-1}\right)$ respecto a los tratamientos con luz LED evaluados, quienes estuvieron en un intervalo de 13.0 y 13.2 ( $\mathrm{mol} \mathrm{m}^{-2} \mathrm{~d}^{-1}$ ) (Tabla 1), ocasionó una mayor síntesis de clorofila a para hacer más eficiente la captura y el uso de la luz, lo cual es un mecanismo de plantas adaptadas a baja intensidad de luz (Qiansheng et al., 2014).

Por otra parte, en relación al resto de pigmentos fotosintéticos evaluados, diversos autores sí encontraron diferencias estadísticas en diversos cultivos cuando se usaron tratamientos con diferentes porcentajes de luz azul y roja, como es el caso de la concentración de clorofila b en plantas de D. purpurea (Verma et al., 2018); en la concentración de clorofila total en plántulas de tomate (Hernández et al., 2016), plántulas de pepino (Hernández y Kubota, 2016), plántulas de colza (Shengxin et al., 2016) y plantas de espinaca (Agarwal et al., 2018); en la relación de clorofila a/b en plántulas de colza (Shengxin et al., 2016) y plantas de espinaca (Agarwal et al., 2018) y finalmente, en la concentración de carotenoides en plantas de Digitalis purpurea (Verma et al., 2018) y plantas de espinaca (Agarwal et al., 2018).

El comportamiento de los pigmentos fotosintéticos que no presentaron diferencias estadísticas, se pudo atribuir al alto grado de plasticidad de la aclimatación que las plantas tuvieron bajo los diferentes tipos de luz (Berkovich et al., 2017; Pocock, 2015), lo cual es consistente con los resultados de las diversas investigaciones que se mencionaron previamente, en donde se obtuvieron diferentes resultados con tratamientos con proporciones de luz LED azul y roja.

\section{CONCLUSIONES}

El tratamiento con luz azul al 100\% promovió el crecimiento del tallo, mientras el testigo con luz fluorescente promovió el crecimiento de la hoja. Los diferentes tratamientos con luz LED no tuvieron efecto en peso seco de parte aérea ni en pigmentos fotosintéticos, por otro lado, los tratamientos que combinaron luz LED azul y roja indujeron un efecto negativo en la altura de plántula, distancia de entrenudos y peso fresco de tallo. 


\section{REFERENCIAS}

Adame-García, J., Murillo-Cuevas, F.D., Flores-de la Rosa, F.R., Velázquez-Mendoza, V., López-Vázquez, M., Cabrera-Mireles, H. y Antonio-Vázquez, E. 2021. Identificación molecular y evaluación de bacterias en el desarrollo vegetativo y producción de chile habanero. Biotecnia. 23(3): 151-157. https://doi.org/10.18633/biotecnia.v23i3.1480

Agarwal, A., Gupta, S.D., Barman, M. y Mitra, A. 2018. Photosynthetic apparatus plays a central role in photosensitive physiological acclimations affecting spinach (Spinacia oleracea L.) growth in response to blue and red photon flux ratios. Environmental and Experimental Botany. 156: 170-182. https://doi.org/10.1016/j. envexpbot.2018.09.009

Amanullah. 2015. Specific leaf area and specific leaf weight in small grain crops wheat, rye, barley, and oats differ at various growth stages and NPK source. Journal of Plant Nutrition. 38: 1694-1708. https://doi.org/10.1080/01904167.2015.101 7051

AOAC. 1980. Official methods of analysis. 12th ed. Association of Official Analytical Chemistry. Washington, D.C.

Berkovich, Y.A., Konovalova, I.O., Smolyanina, S.O., Erokhin, A.N., Avercheva, O.V., Bassarskaya, E.M., Kochetova, G.V., Zhigalova, T.V., Yakovleva, O.S. y Tarakanov, I.G. 2017. LED crop illumination inside space greenhouses. REACH Reviews in Human Space Exploration. 6: 11-24. http://dx.doi. org/10.1016/j.reach.2017.06.001

Bojórquez-Quintal, E., Velarde-Buendía, A., Ku-González, A., Carillo-Pech, M., Ortega-Camacho, D., Echevarría-Machado, I., Pottosin I. y Martínez-Estévez, M. 2014. Mechanisms of salt tolerance in habanero pepper plants (Capsicum chinense Jacq.): proline accumulation, ions dynamics and sodium root-shoot partition and compartmentation. Frontiers in Plant Science. 5: 605. http://dx.doi.org/10.3389/ fpls.2014.00605

Carpita, N.C., Maureen, J.R. y McCann, C. 2015. The Cell Wall. En: Biochemistry and Molecular Biology of Plants. B. B. Buchanan, W. Gruissem y R.L. Jones (eds.), pp 45-110. Wiley Blackwell, UK.

Castillo-Aguilar, C. de la C., Quej-Ch, I. V., Coh-Méndez, D., Carrillo-Ávila, E. y Monsalvo-Espinosa, A. 2015. Producción de planta de chile habanero (Capsicum chinense Jacq.). Agro-Productividad. 8(4): 73-78.

CICY. 2016. Ficha informativa unidad productora de semillas de chile habanero. Centro de Investigación Científica de Yucatán. Abril (2016): 1-15. [Consultado 10 enero 2020]. Disponible en: https://www.cicy.mx/Documentos/CICY/ quienes-somos/2016/Ficha-UPS-Habanero.pdf

Cosgrove, D. J. 2005. Growth of the plant cell wall. Nature Reviews Molecular Cell Biology. 6(11): 850-861. https://doi. org/10.1038/nrm1746

Demotes-Mainard, S., Pérona, T., Corotb, A., Bertheloota, J., Gourrierecb, J.L., Pelleschi-Travierb, S., Crespel, L., Morel, P., Huché-Thélier, L., Boumaza, R., Vian, A., Guérin, V., Leduc, N. y Sakr, S. 2016. Plant responses to red and far-red lights, applications in horticulture. Environmental and Experimental Botany. 121: 4-21. http://dx.doi.org/10.1016/j. envexpbot.2015.05.010

Engels, C., Kirkby, E. y White, P. 2012. Mineral Nutrition, Yield and Source-Sink Relationships. En: Marschner's Mineral Nutrition of Higher Plants. P. Marschner (ed.), pp 85-133.
Academic Press, USA. https://dx.doi.org/10.1016/B978-012-384905-2.00005-4

Hernández, R. y Kubota, C. 2016. Physiological responses of cucumber seedlings under different blue and red photon flux ratios using LEDs. Environmental and Experimental Botany. 121: 66-74. http://dx.doi.org/10.1016/j. envexpbot.2015.04.001

Hernández, R., Eguchib, T., Devecic, M. y Kubota, C. 2016. Tomato seedling physiological responses under different percentages of blue and red photon flux ratios using LEDs and cool white fluorescent lamps. Scientia Horticulturae. 213: 270-280. http://dx.doi.org/10.1016/j.scienta.2016.11.005

Hoagland, D.R. y Arnon, D.I. 1950. The water-culture method for growing plants without soil. Circular 347. California Agricultural Experiment Station. California, USA. 32 p.

Huché-Thélier, L., Crespel, L., Gourrierec, J.L., Morel, P., Sakr, S. y Leduc, N. 2016. Light signaling and plant responses to blue and UV radiations-Perspectives for applications in horticulture. Environmental and Experimental Botany. 121: 22-38. https://dx.doi.org/10.1016/j.envexpbot.2015.06.009

Katagiri, F., Canelon-Suarez, D., Griffin, K., Petersen, J., Meyer, R.K. y Siegle, M. 2015. Design and construction of an inexpensive homemade plant growth chamber. PLoS ONE. 10(5): e0126826. https://doi.org/10.1371/journal.pone.0126826

Kim, H.M., y Hwang, S.J. 2019. The growth and development of 'Mini Chal' tomato plug seedlings grown under monochromatic or combined red and blue light-emitting diode. Horticultural Science and Technology. 37(2): 190-205. https://doi.org/10.12972/kjhst.20190019

Kozai, T., Shibuya T., He, D., Zobayed, S. y Chun, C. 2016. Transplant production in closed system. En: Plant factory: An indoor vertical farming system for efficient quality food production. T. Kozai, G. Niu y M. Takagaki (eds.), pp 237-269. Academic Press, USA. https://dx.doi.org/10.1016/B978-0-12801775-3.00019-6

Kozai, T. y Niu, G. 2016. Role of the plant factory with artificial lighting (PFAL) in urban areas. En: Plant factory: An indoor vertical farming system for efficient quality food production. T. Kozai, G. Niu y M. Takagaki (eds.), pp 115-128. Academic Press, USA. https://dx.doi.org/10.1016/B978-0-12-8017753.00002-0

Kubota, C. 2016. Growth, development, transpiration and translocation as affected by abiotic environmental factors. En: Plant factory: An indoor vertical farming system for efficient quality food production. T. Kozai, G. Niu y M. Takagaki (ed.), pp 151-164. Academic Press, USA. http:// dx.doi.org/10.1016/B978-0-12-801775-3.00010-X

Lim, T. K. 2013. Capsicum chinense. En: Edible medicinal and nonmedicinal plants: Volume 6, Fruits. T. K. Lim (ed.), pp 205-212. Springer Science+Business Media, Dordrecht. https://doi. org/10.1007/978-94-007-5628-1_30

Liu, X. Y., Jiao, X. L., Chang, T. T., Guo, S. R. y Xu, Z. G. 2018. Photosynthesis and leaf development of cherry tomato seedlings under different LED-based blue and red photon flux ratios. Photosynthetica. 56: 1212-1217. https://dx.doi. org/10.1007/s11099-018-0814-8

Mendoza-Paredes, J.E., Castillo-González, A.M., Avitia-García, E., Valdéz-Aguilar, L.A. y García-Mateos, M.R. 2021a. Efecto de diferentes proporciones de luz LED azul:roja en plantas de chile habanero (Capsicum chinense Jacq.). Biotecnia. 23(1): 110-119. https://doi.org/10.18633/biotecnia.v23i1.1288 
Mendoza-Paredes, J.E., Castillo-González, A.M., Valdéz-Aguilar, L.A., Avitia-García, E. y García-Mateos, M.R. 2021b. Respuesta de cilantro (Coriandrum sativum L.) a la luz LED azul y roja. Biotecnia. 23(2): 149-160. https://doi.org/10.18633/ biotecnia.v23i2.1340

Pocock, T. 2015. Light-emitting diodes and the modulation of specialty crops: light sensing and signaling networks in plants. HortScience. 50(9): 1281-1284. https://dx.doi. org/10.21273/HORTSCI.50.9.1281

Poiré, R., Wiese-Klinkenberg, A., Parent, B., Mielewczik, M., Schurr, U., Tardieu, F., y Walter, A. 2010. Diel time-courses of leaf growth in monocot and dicot species: endogenous rhythms and temperature effect. Journal of Experimental Botany. 61: 1751-1759. https://doi.org/10.1093/jxb/erq049

Qiansheng, L., Deng, M., Xiong, Y., Coombes, A. y Zhao, W. 2014. Morphological and photosynthetic response to high and low Irradiance of Aeschynanthus longicaulis. The Scientific World Journal. 2014: 347461. 1-8, http://dx.doi. org/10.1155/2014/347461

Ruiz-Lau, N., Medina, F. y Martínez, M. 2011, El chile habanero: su origen y usos. Ciencia. Julio-Septiembre: 70-77.

SAS Institute. 2002. SAS System for Windows Computer Program. Software Version 9.0. Cary, North Carolina, USA. 1861 p.

Shengxin, C., Chunxia, L., Xuyang, Y., Song, C., Xuelei, J., Xiaoying, L., Zhigang, X. y Rongzhan, G. 2016. Morphological, photosynthetic, and physiological responses of rapeseed leaf to different combinations of red and blue lights at the rosette stage. Frontiers in Plant Science. 7: 1144. https://doi. org/10.3389/fpls.2016.01144
Stanton, K.M., Weeks, S.S., Dana, M.N. y Mickelbart, M.V. 2010. Light exposure and shade effects on growth, flowering, and leaf morphology of Spiraea alba Du Roi and Spiraea tomentosa L. HortScience. 45(12): 1912-1916. https://doi. org/10.21273/HORTSCI.45.12.1912

Tsukaya, H. 2005 Leaf shape: genetic controls and environmental factors. The International Journal of Developmental Biology. 49: 547-555. https://doi.org/10.1387/ijdb.041921ht

Trujillo, J.J.G. 2018. Caracterización de los recursos genéticos de Capsicum chinense Jacq. en la península de Yucatán. En: Mejoramiento genético del chile habanero de la Península de Yucatán. T.A. González (ed.), pp 37-43. Centro de Investigación Científica de Yucatán. Mérida, México.

Verma, S.K., Gantait, S., Jeong, B.R. y Hwang, S.J. 2018. Enhanced growth and cardenolides production in Digitalis purpurea under the influence of different LED exposures in the plant factory. Nature Scientific Reports. 8: 18009. https://doi. org/10.1038/s41598-018-36113-9

Walter, A., Silk, W.K. y Schurr, U. 2009. Environmental effects on spatial and temporal patterns of leaf and root growth. Annual Review of Plant Biology. 60: 279-304. https://doi. org/10.1146/annurev.arplant.59.032607.092819

Wollaeger, H.M. y Runkle, E.S. 2015. Growth and acclimation of impatiens, salvia, petunia, and tomato seedlings to blue and red light. HortScience. 50(4): 522-529. https://doi. org/10.21273/HORTSCI.50.4.522

Yamori, W. 2016. Photosynthesis and respiration. En: Plant factory: An indoor vertical farming system for efficient quality food production. T. Kozai, G. Niu y M. Takagaki (ed.), pp 141-149. Academic Press, USA. https://dx.doi. org/10.1016/B978-0-12-801775-3.0009-3(LAI). 